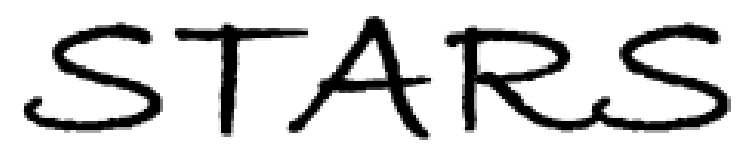

University of Central Florida

STARS

$1-1-2006$

\title{
Designing triple resonance Tesla transformers of arbitrary modal frequency ratio
}

John Randolph Reed

University of Central Florida

Find similar works at: https://stars.library.ucf.edu/facultybib2000 University of Central Florida Libraries http://library.ucf.edu

This Article is brought to you for free and open access by the Faculty Bibliography at STARS. It has been accepted for inclusion in Faculty Bibliography 2000 s by an authorized administrator of STARS. For more information, please contactSTARS@ucf.edu.

\section{Recommended Citation}

Reed, John Randolph, "Designing triple resonance Tesla transformers of arbitrary modal frequency ratio" (2006). Faculty Bibliography 2000s. 6494.

https://stars.library.ucf.edu/facultybib2000/6494

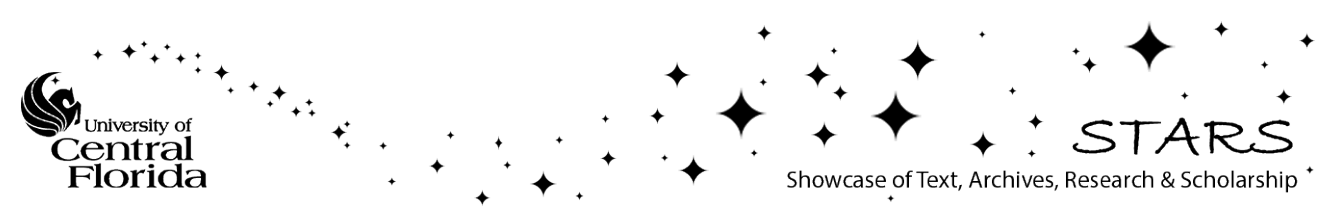




\section{Designing triple resonance Tesla transformers of arbitrary modal frequency ratio}

Cite as: Rev. Sci. Instrum. 77, 033301 (2006); https://doi.org/10.1063/1.2173949

Submitted: 01 November 2005 . Accepted: 19 January 2006 . Published Online: 10 March 2006

John Randolph Reed

\section{ARTICLES YOU MAY BE INTERESTED IN}

Analytical expression for the output voltage of the triple resonance Tesla transformer Review of Scientific Instruments 76, 104702 (2005); https://doi.org/10.1063/1.2093764

Optimal performance for Tesla transformers

Review of Scientific Instruments 73, 3332 (2002); https://doi.org/10.1063/1.1498905

A Tesla transformer high-voltage generator

Review of Scientific Instruments 46, 1 (1975); https://doi.org/10.1063/1.1134057

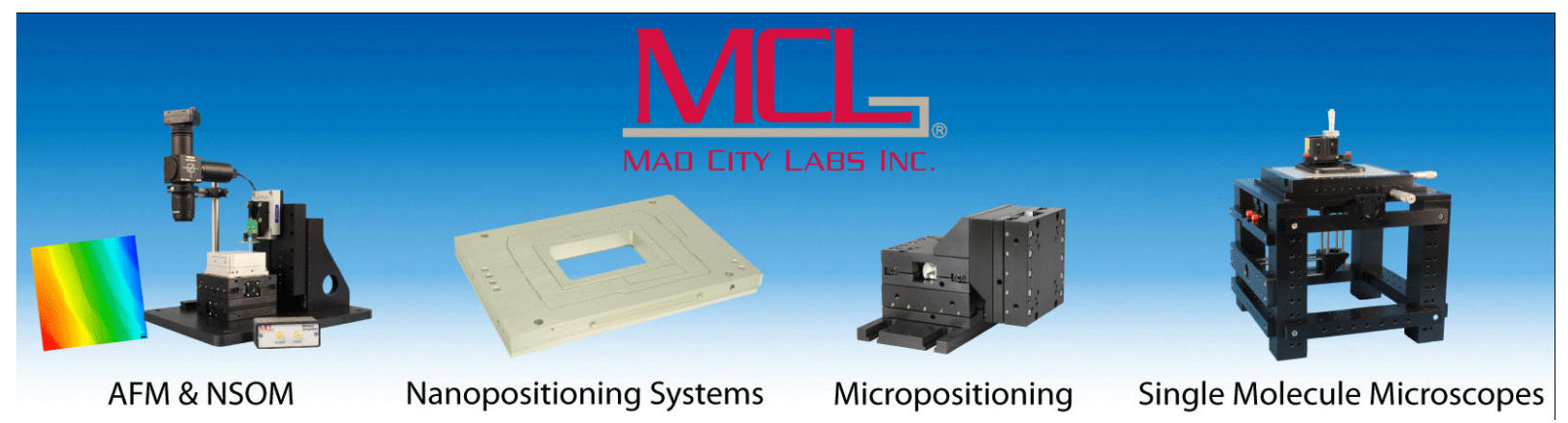




\title{
Designing triple resonance Tesla transformers of arbitrary modal frequency ratio
}

\author{
John Randolph Reed \\ Department of Engineering and Computer Science, The University of Central Florida, \\ 400 Central Florida Boulevard, Orlando, Florida 32816
}

(Received 1 November 2005; accepted 19 January 2006; published online 10 March 2006)

\begin{abstract}
The purpose of this article is to disclose an automated method to design and investigate multimegavolt triple resonance Tesla transformers. The pulse transformer's "frequency equation" is presented for the first time. The frequency equation derivation properly models all the inductors, with their self-capacitances, which have yet to be treated in an orthodox manner. The analysis gives new insight into the transformer by showing the relationship between the roots of the frequency equation and the transformer's modal frequencies. The roots are shown to be subject to manipulation, and so the modal frequencies are controllable. The method efficiently extracts solutions (transformer circuits) from the frequency equation constrained to oscillate at an arbitrary and general modal frequency ratio (to include noninteger). A ratio of the present general interest is 1:2:3. This particular ratio forces the maxima of the three coexisting modal oscillations to align, and their amplitudes sum to produce a local maximum, at a specific time. The same alignment phenomenon occurs with the dual resonance transformer with a modal ratio of 1:2. A pulse transformer is designed as a demonstration. The energy in each of the three oscillations is examined at the moment of peak voltage in the demonstration transformer to show the investigative power of the new equations. This generalized tool will prove useful in the campaign to analytically locate global maximums from the triple resonance transformer's governing amplitude equation for output voltage. () 2006 American Institute of Physics. [DOI: 10.1063/1.2173949]
\end{abstract}

\section{INTRODUCTION AND PREVIOUS WORK}

The well-understood Tesla transformer is the "dual resonance" device (possessing two coupled parallel $L C$ circuits and two modal frequencies). The dual resonance transformer has been used as a pulsed power supply for various industrial applications including dielectric testing, directed energy investigations, and the production of intense electron beams ${ }^{1,2}$ for high-energy research. An important feature of this pulse transformer is it requires only one switch.

The more obscure configuration of the transformer is the "triple resonance" device (possessing three parallel $L C$ circuits and three modal frequencies). Tesla also invented this form of pulse transformer and obtained a patent ${ }^{3}$ on the same in 1914 (submitted 1902). The advantage is the higher pulse powers by only adding a properly sized third inductor. Figure 1, of Ref. 12, shows a schematic of a typical transformer with its circuit parameters. It is interesting to note that Tesla stated that "...the three waves must fall together." And by the experiment accomplished the same using a rotating mirror in stroboscopic relationship with the spark-gap switch before 1899.

In the past the triple resonance transformers were constructed experimentally, because the device was very poorly understood. There were no physical models that comprehensively described the relationship of the frequency and amplitude of the modal oscillations of the circuit relative to its eight degrees of freedom.

Bieniosek $^{4,5}$ conducted additional investigations into a three modal frequency transformer whose design departed from Tesla's original configuration. Bieniosek advocated restricted modal frequencies in the ratio of specific whole numbers $(1: 2: 3)$ and external capacitors as necessary to obtain operability. Bieniosek added extra capacitors when all previous transformers, as shown in Fig. 1, have been built without any external capacitors, and operated at the multimegavolt level [some at $13 \mathrm{MV}$ (Ref. 6)]. The self-capacitances of the Tesla transformer of Fig. 1 are intrinsic to its inductors such as the turn-to-turn winding capacity. These inductors withstand the axial high-voltage stress due to the turn-to-turn voltage grading, and their large radii of curvature increase their radial electrostatic insulation. Additionally, the magnetically coupled inductors with large radii of curvature made higher mutual inductances, $M$, easier to obtain.

Bieniosek's design claims to transfer, with perfect efficiency, $100 \%$ of the energy from the transformer to the load (e.g., no energy remains behind; trapped anywhere), therefore resulting in higher voltage gains and more useful pulse power.

Historically and correctly, $100 \%$ energy transfer simply meant that the primary energy storage capacitor was totally discharged the instant the output voltage peaked in the secondary circuit. This definition was actually an explanation of how the dual resonance transformer reached its extreme pulse powers. The energy from the relatively large primary capacitance was transferred to the small self-capacitance of the secondary inductor in series with the electrostatic capacitance of the electron gun's "corona shield." Because the energy transfer in the device is efficient $[\sim 90 \%$ (Ref. 9)] the 


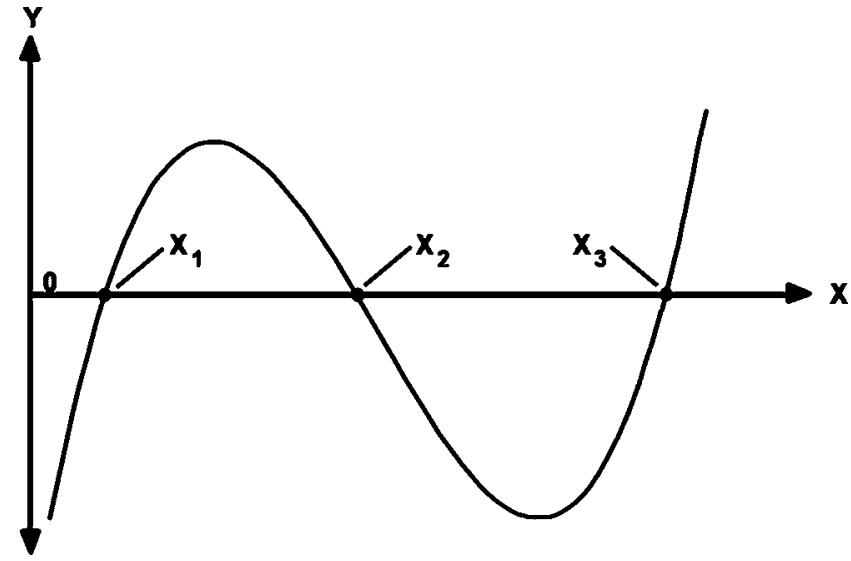

FIG. 1. Plot of the "frequency squared" cubic $y=x^{3}-40 x^{2}+411 x-875$. $=0$. Note, the roots $x_{1}, x_{2}$, and $x_{3}$ have the relationship $x_{2}=4 x_{1}$, and $x_{3}=9 x_{1}$. The roots were located with a hand-held pointer. The numerical value of the roots are $x_{1}=\omega_{1}^{2}=2.899, x_{2}=\omega_{2}^{2}=11.595$, and $x_{3}=\omega_{3}^{2}=26.102$. The frequencies in Hertz are found from the roots by the equation, $f_{i}=\left\{\left(x_{i}\right)^{1 / 2} /(2 \pi)\right\} 10^{6}$, with $i=1-3$. Multiplication by $10^{6}$, to obtain Hertz, is necessary as the working units chosen are microfarad and microhenry. Therefore, $f_{1}=270911, f_{2}=541948$, and $f_{3}=813135$.

voltage dramatically increases when the energy is transferred into the small secondary capacitance. Some energy is trapped in the self-capacitance of the transformer, such as a portion in the turn-to-turn capacitance, and the parasitic coupling into the surroundings.

Bieniosek ${ }^{7}$ later patented his transformer circuit parameter values. The patent also shows a circuit simulator analysis predicting a zero voltage between the second and third inductors and ground, when the output voltage peaks. The circuit simulator plots are shown in the patent to reinforce the correctness of his claims.

Bieniosek's design begs revisitation because to accomplish his ideal transfer conventional capacitors are employed across multimegavolt potentials. This impracticable fact goes unrecognized, but will be pointed out and discussed in the next paragraph.

Later, de Queiroz ${ }^{8}$ reworked Bieniosek's model and again applied the erroneous design of perfect energy transfers to the triple resonance Tesla-transformer circuit. References $4,5,7$, and 8 show conventional capacitors supporting multimegavolt stresses in both author's designs. This can easily be seen by examining Sec. 25 of Bieniosek's patent ${ }^{7}$ which "...introduces an external capacitance in parallel with the internal capacitance of the transformer." There is typically several megavolts across the transformer at this point.

How these enormous stresses in the external capacitors were to be negotiated was never discussed in any of their expositions.

Bieniosek and de Queiroz also assumed that the selfcapacitance of the third inductor can be "lumped" with the capacitance of the load to mathematically simplify the problem. These self-capacitances (internal) are in series and cannot be lumped or ignored, as they are the actual underpinning of the operation of the device. Each inductor must be allowed to oscillate (cyclic storage of energy alternately from static and dynamic forms) in order to transfer energy through the device. An accurate mathematical analysis can only be performed if each inductor is properly treated as a distinct $L C$ oscillator. The authors locally zero the self-capacitance of the third inductor by moving it downstream, and simply enlarging the load capacitance the proper amount. A circuit simulator [e.g., PSPICE (Ref. 9)] will show misleading results stemming from this incorrect input data. If the selfcapacitance of the third inductor is zeroed, energy cannot be stored statically in the same, when the output voltage peaks. The simulator is forced to show a voltage of zero between ground and the second and third self-capacitances at that instant. This result, under a cursory examination, will give the appearance of no energy being captured by the inductor's self-capacity, and mistakenly interpreted as perfect energy transfer through the device, and yield larger gains. There are voltage plots in both Bieniosek's patent and de Queiroz's paper, showing zero potential between the transmission line and ground at the instant the voltage on $C_{4}$ peaks. Later, in this analysis, a PSPICE examination will show the trapped energy within the device.

\section{DYNAMIC CIRCUIT EQUATIONS OF THE TRANSFORMER}

The system of dynamic circuit equations of Fig. 1 used to derive the transformer's frequency equation is

$$
\begin{aligned}
& V_{1}+L_{1} C_{1} V^{\prime \prime}{ }_{1}+M\left(C_{2}+C_{4}\right) V_{2}^{\prime \prime}+M C_{4} V_{3}{ }_{3}=0, \\
& V_{2}+M C_{1} V_{1}{ }_{1}+L_{2}\left(C_{2}+C_{4}\right) V_{2}^{\prime \prime}{ }_{2}+L_{2} C_{4} V_{3}{ }_{3}=0, \\
& V_{3}+0+L_{3} C_{4} V_{2}^{\prime \prime}{ }_{2}+L_{3}\left(C_{3}+C_{4}\right) V_{3}^{\prime \prime}{ }_{3}=0 .
\end{aligned}
$$

The variable definitions and complete derivation of the system of Eq. (1), including the use of the relation $V_{4}=V_{2}+V_{3}$, are on deposit as supplemental information in EPAPS. ${ }^{10}$

\section{CIRCUIT DESIGN USING CONSTRAINED FREQUENCY EQUATIONS}

In previous transformer design a significant difficulty arises; that being, the frequency ratio of interest must be obtained by the sizing of the circuit parameters, but the resulting parameters must also produce a useful voltage gain. In other words, circuit parameters can be found experimentally that obtain the frequency ratio, but the resulting voltage gain will be uselessly low. ${ }^{8}$

This section discusses and demonstrates the use of the frequency equation to obtain values of circuit components to produce a manifold of transformers with a specified modal frequency ratio. The investigator is in no way limited in the choice of frequency ratio.

The investigator can quickly examine the performance of each transformer and find one, or more, that fit his interest.

The frequency ratio is not the only parameter under the control of the investigator but also all the other circuit parameters. 
TABLE I. Solution data for the example triple resonance transformer. This transformer was designed using the frequency equation for the triple resonance transformer circuit. The modal frequencies in the coupled system, $f_{i}$, where $i=1-3$, are in the ratio of $1: 2: 3$.

\begin{tabular}{ccc}
\hline \hline Constant & Value & Unit \\
\hline$C_{1}$ & 0.299 & $\mu \mathrm{F}$ \\
$C_{2}$ & 0.0001575 & $\mu \mathrm{F}$ \\
$C_{3}$ & 0.0000255 & $\mu \mathrm{F}$ \\
$C_{4}$ & 0.000060 & $\mu \mathrm{F}$ \\
$L_{1}$ & 0.87 & $\mu \mathrm{H}$ \\
$L_{2}$ & 639.9 & $\mu \mathrm{H}$ \\
$L_{3}$ & 820.0 & $\mu \mathrm{H}$ \\
$k$ & 0.666 & $\mathrm{~Hz}$ \\
$f_{1}$ & 270911,270890, and 270991 & $\mathrm{~Hz}$ \\
$f_{2}$ & 541822,541780, and 541948 & $\mathrm{~Hz}$ \\
$f_{3}$ & 812733,812670, and 813135 & \\
Gain & $\sim 50: 1$ & \\
\hline \hline
\end{tabular}

It is now emphasized that a numerical optimizer solves the constrained frequency equation, and extracts the values of the circuit parameters found to produce the specified frequency ratio.

The function of the optimizer is to find the minimum of a bounded multivariable function without having to determine the derivatives. This technique is used when obtaining the derivatives and is extremely difficult or impossible. One should not conclude that optimal transformers are being designed because a numerical optimizer is used to satisfy a constrained frequency equation. Transformers are being found with the modal frequency ratio and some of the circuit parameters requested by the investigator.

The transformer design program, also on deposit in EPAPS,${ }^{10}$ is exercised once the modal frequency ratio is selected, and each circuit parameter is "bounded" or fixed. The electrostatic capacitance of the electron gun's corona shield, $C_{4}$, is an example of a "fixed" circuit parameter. The frequency ratio can be noninteger, for example, 1:1.5:2.7.

The optimizer varies the values of the parameters, in "parameter space," to minimize the constrained frequency equation, and thereby extracts a solution with modal frequencies as specified.

Jacob $^{11}$ developed the optimizer. The transformer design program $^{10}$ produces an example circuit with a voltage gain of 50:1 (shown in Table I). A gain of 40 is generally considered large. None of the resulting parameters are physically unrealistic or difficult to obtain in the $\sim 50: 1$ device.

The design technique is as follows: the transformer program of Ref. 10 and a program containing the closed form solution $^{12}$ for the transformer's output voltage are employed in an investigative manner to derive a manifold of transformers; and then search the manifold for one of the suitable performance.

The task of generating a manifold of circuits is made possible, because the frequency equation is restricted to only deliver circuits that produce the specified modal frequency ratio.

The optimizer displaces each parameter of the frequency equation thousands of times to move about the specified pa- rameter space. A better gain, at the frequency ratio, may sometimes be found by inputting the final results for the initial conditions for a repeated run, and then displace one parameter toward its upper boundary. The "toggling" of the optimizer's initial search position, in this manner, has led to desirable results.

\section{WRITING CONSTRAINED FREQUENCY EQUATIONS}

The sixth order frequency equation for the high- $Q$ triple resonance circuit of Fig. 1 is

$$
\begin{aligned}
\{(1- & \left.\left.k^{2}\right) L_{1} L_{2} L_{3} C_{1}\left[C_{2} C_{3}+C_{4}\left(C_{2}+C_{3}\right)\right]\right\} \omega^{6} \\
& -\left\{\left(1-k^{2}\right) L_{1} L_{2} C_{1}\left(C_{2}+C_{4}\right)+L_{2} L_{3}\left[C_{2} C_{3}\right.\right. \\
& \left.\left.+C_{4}\left(C_{2}+C_{3}\right)\right]+L_{3} L_{1} C_{1}\left(C_{3}+C_{4}\right)\right\} \omega^{4} \\
& +\left\{L_{1} C_{1}+L_{2}\left(C_{2}+C_{4}\right)+L_{3}\left(C_{3}+C_{4}\right)\right\} \omega^{2}-1=0 .
\end{aligned}
$$

The above frequency equation, derived and deposited in EPAPS, ${ }^{10}$ describes all the possible free oscillations the circuit of Fig. 1 can display, depending upon the value of its parameters.

The frequency equation will later be considered a cubic equation in $\omega^{2}$. This technique is well known in vibration analysis. The cubic in $\omega^{2}$ has been referred to the "frequency squared equation," or the "z equation.",13

Denote the coefficient of $\omega^{6}$ as $A$, the coefficient of $\omega^{4}$ as $B$, and the coefficient of $\omega^{2}$ as $C . A>0, B>0$, and $C>0$ is assumed when writing Eq. (2). The desired frequency ratio is guaranteed if a specific constraining condition between $B$ and $C$ and a separate specific constraining condition between $A$ and $C$ are concurrently satisfied, expressed functionally as

$$
u(B, C)=0 \text { and } v(A, C)=0 .
$$

A single constraint equation is formed by squaring both conditions and adding them, expressed functionally again as

$$
\mathrm{FU}=[u(B, C)]^{2}+[v(A, C)]^{2}=0 .
$$

The constraint is equal to FU, which is its label in the computer program (see subroutine FN) of Ref. 10. The optimizer searches within the bounds (supplied by the investigator) of the electrical component values (parameter space) comprising the coefficients $A, B$, and $C$, and extracts a combination of values that minimizes FU. The algebraic structure of the constraint, Eq. (4), keeps the search mechanism from oscillating about zero by keeping FU positive. Upon obtaining an FU very near zero, a value on the order of $10^{-40}$ is reasonably strong; the constraint equation is satisfied. The resulting electrical components will produce a circuit with the chosen frequency ratio. The functional value of FU must be very small due to the repeated arithmetic of the relatively small and large numbers that describe this type of electrical system.

The resulting value of the coefficients $(A, B$, and $C)$ will later be shown to predict the fundamental modal frequency. As an example, the coefficient $C$ yields the fundamental mode in Hertz as: 


$$
f_{1} \approx\left(10^{6} / 2 \pi\right)\left[(1.166574219 \cdots) / C^{1 / 2}\right] \mathrm{Hz} .
$$

Equation (5) is multiplied by $10^{6}$ to obtain Hertz as microhenry and microfarad were chosen for working units in the transformer design program ${ }^{10}$ for aiding arithmetic accuracy. Equation (5) will be further detailed later in the analysis.

The fundamental mode can also be found using a combination of coefficients $A$ and $B$, in the same manner, as was shown in Eq. (5).

The derivation of the constraint equation is now given to show how the frequency equation is limited to deliver solutions that only produce the specified modal frequency ratio.

The angular modal frequencies are chosen to have the relationship 1:2:3, or $\omega_{2} / \omega_{1}=2$ and $\omega_{3} / \omega_{1}=3$. Therefore let $\left(\omega_{2} / \omega_{1}\right)^{2}=x_{2} / x_{1}=4$ and $\left(\omega_{3} / \omega_{1}\right)^{2}=x_{3} / x_{1}=9$, or $x_{2}=4 x_{1}$ and $x_{3}=9 x_{1}$.

If a different modal frequency ratio is desired, then the preceeeding relationships would have to be changed accordingly. Writing Eq. (2) in the frequency squared form and using a property of cubics ${ }^{14}$ yields

$$
\begin{aligned}
y & =x^{3}-(B / A) x^{2}+(C / A) x-(1 / A) \\
& =\left(x-x_{1}\right)\left(x-x_{2}\right)\left(x-x_{3}\right) .
\end{aligned}
$$

The subscripted $x$ 's are the three real and different roots of the cubic, which were seen earlier in connection with the frequency ratio. Each crossing of the $x$ axis, by the cubic, corresponds to the square of an angular modal frequency. Substitution of the roots into Eq. (6) after some algebraic manipulation yields

$$
y=x^{3}-14 x_{1} x^{2}+49 x_{1}{ }^{2} x-36 x_{1}^{3} .
$$

The square of the fundamental angular frequency $x_{1}=\omega_{1}^{2}$ is found using Eqs. (6) and (7) as

$$
14 x_{1}=B / A \text { or } x_{1}=B /(14 A) \text {. }
$$

The constraints on the coefficients $(A, B$, and $C)$ of the frequency equation to obtain the frequency ratio is found using Eqs. (6)-(8) yielding

$$
x_{1}{ }^{2}=C /(49 A) \text { or }\left[49 /(14)^{2}\right](B / A)^{2}=C / A, \quad B^{2}=4 A C
$$

and

$$
\begin{aligned}
& x_{1}{ }^{3}=1 /(36 A) \text { or }\left[36 /(14)^{3}\right](B / A)^{3}=1 / A, \\
& B^{3}=76.22 \cdots A^{2} .
\end{aligned}
$$

A constraint between $B$ and $C$, and $A$ and $C$ can be obtained, in integers, by manipulating Eqs. (9) and (10) to yield

$$
B=(72 / 343) C^{2} \text { and } A=(1296 / 117649) C^{3} .
$$

A constraint having the form given by Eq. (4) is written, using Eq. (11) as

$$
\begin{aligned}
\mathrm{FU}= & \left(A-0.011015818 \cdots C^{3}\right)^{2} \\
& +\left(B-0.209912536 \cdots C^{2}\right)^{2}=0.0 .
\end{aligned}
$$

The optimizer satisfies Eq. (12), along with its bounded and fixed parameters, and the resulting values of $A, B$, and $C$ in this case are

$$
\begin{aligned}
& A=0.0011419662137 \ldots, \\
& B=0.046322974941 \ldots,
\end{aligned}
$$

and

$$
C=0.4697632850020 \ldots .
$$

The usefulness of the optimizer can be appreciated by inspecting the coefficients of the frequency equation $(A, B$, and $C$ ) and observing that $A$ has 10 parameters, $B$ has 18 parameters, and $C$ has 8 parameters. The parameters appear as products, and products of sums, and when installed in the constraint equation are raised to various powers. The optimizer is promptly able to satisfy constraint, Eq. (12), and this example drove $\mathrm{FU}$ to $10^{-50}$.

Equation (8), the square of the fundamental angular modal frequency is

$$
\omega_{1}^{2} \text { or } 4 \pi^{2} f_{1}^{2} \approx B /(14 A)=2.899 \ldots,
$$

and the fundamental modal frequency, $f_{1}$, in Hertz, as stated in Eq. (5), is

$$
\begin{aligned}
f_{1} \approx & \left(10^{6} / 2 \pi\right)\left[(1.166574219 \cdots) / C^{1 / 2}\right] \mathrm{Hz} \\
& \text { or } 270890 \mathrm{~Hz} .
\end{aligned}
$$

Multiplication by $10^{6}$ is necessary to obtain Hertz as stated before. Once $f_{1}$ has been computed, the other two frequencies are

$$
f_{2} \approx 2 f_{1} \text { and } f_{3} \approx 3 f_{1} .
$$

Other coefficients can be used to obtain the fundamental modal frequency. This is understood by using Eq. (13), which yields

$$
f_{1} \approx\left(10^{6} / 2 \pi\right)[B /(14 A)]^{1 / 2} \text { or } 270911 \mathrm{~Hz} .
$$

The other two frequencies are found simply by the relationships (15).

Furthermore, if desired, a root finder can be used on polynomial (6) and the values of coefficients $A, B$, and $C$, found by the optimizer, to obtain the roots $x_{1}, x_{2}$, and $x_{3}$. The modal frequencies are related to the positive roots as discussed before as

$$
\omega_{1}=\left(x_{1}\right)^{1 / 2}, \quad \omega_{2}=\left(x_{2}\right)^{1 / 2}, \quad \text { and } \quad \omega_{3}=\left(x_{3}\right)^{1 / 2} .
$$

The closed form solution, of Ref. 12, for the transformers output voltage also gives the modal frequencies, which are as follows:

$$
f_{1} \approx 270991 \mathrm{~Hz}, f_{2} \approx 541948 \mathrm{~Hz}
$$

and

$$
f_{3} \approx 813135 \mathrm{~Hz} \text {. }
$$

\section{RESULTS}

The four goals of this exercise were to use the method to find a transformer with a modal frequency ratio of 1:2:3, a large primary capacitor $C_{1}, C_{4}$ at a fixed value, and necessarily, the device must possess a useful voltage gain. Also, the circuit components obtained to produce these results must be physically reasonable. 
Bounded starting values for the parameters (electrical components) were first assigned. When the transformer program was running; FU equal to $0.205 \times 10^{-50}$ was obtained along with the circuit data listed in Table I after 1123 trials or manipulations. The wall clock time for the 1123 manipulations was less than $5 \mathrm{~s}$ using a $1.1 \mathrm{GHz}$ processor. The small value of FU indicated that the resulting parameters, composing the coefficients, fulfilled the constraining condition, Eq. (12), and the 1:2:3 frequency ratio is guaranteed.

Due to the arithmetical nature of the problem high numerical precision of the computer is important, and extremely small values of FU are necessary. The transformer design program ${ }^{10}$ was exercised in quadruple precision arithmetic, and the closed form solution program ${ }^{12}$ was exercised in single precision (to show that it can also obtain roots). The computational difficulty lie in the arithmetic of extremely small numbers in combination with large numbers. Secondly, the mathematical nature of the optimizer's search process will not produce exact results. ${ }^{11}$ One should be aware of these computational matters; but for practical purposes they cause no harm, if treated with precautions ordinary to numerical analysis.

Table I contains the frequency ratio and circuit parameters resulting from the analysis. ${ }^{10,12}$ The left column is the frequencies resulting from using Eq. (16) to compute the fundamental. The middle column results from using Eq. (14) to find the fundamental. The right column is the frequencies found by closed form solution ${ }^{12}$ for the transformer's output voltage.

Although frequency ratio was a main target of this analysis; it was found that the primary storage capacitance could be displaced toward its upper boundary and the optimizer repeatedly kept satisfying the constraint equation with everlarger primary capacitors. The end result of this toggling was a large primary storage capacity, which was one of the goals. A gain of 50 was achieved even though some energy remained trapped in the stray capacitances of the transformer. The output pulse reaches its maximum voltage in the first cycle; which is exceedingly desirable, as continued oscillation of the primary capacitor will lead to its eventual destruction.

High magnetic coupling for a compact design can be achieved through the use of a proper ferrite to link the flux and minimize stray inductance. Crushed "ferroxecube" mixed with a suitable wax makes a good core material to enhance performance.

The cubic, equation (6), was plotted for demonstration. The plot of the cubic and the numerical values of its roots are shown in Fig. 1. It is through the cubic's roots the investigator obtains additional insight into the relationship between the circuit parameters and the modal frequencies.

The oscillatory response of the newly found transformer circuit is shown in Fig. 2. The circuit, found from the frequency equation, was inputed to ORCAD PSPICE (Ref. 9) to obtain a plot of the time-dependent voltage between $C_{2}$ and $C_{3}$ (or the voltage between the transmission line and ground) and across the load capacitance, $C_{4}$ (or the output voltage relative to ground).

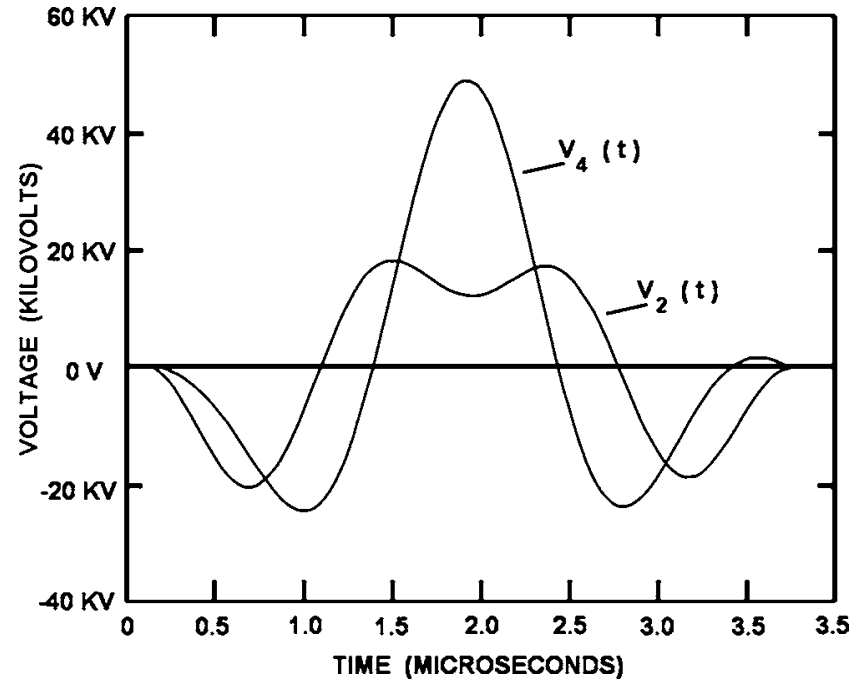

FIG. 2. The transformer's circuit response built with parameters found through the constrained frequency equation. The data in Table I are the inputs to ORCAD PSPICE. $V_{2}(t)$ is the time-dependent voltage of the transmission line relative to ground. The transient plot now shows the energy stored in the self-capacitances when the voltage peaks. $V_{4}(t)$ is the time-dependent voltage across the load capacitance (output voltage). The voltage peaks in the circuit at $50 \mathrm{kV}$. The initial voltage of the primary capacitor is $1 \mathrm{kV}$, so the voltage gain is 50 .

The plot of the output pulse computed by the closed form solution for the output voltage is not included in Fig. 2 as such a plot already exists. ${ }^{12}$

\section{DISCUSSION}

A new and useful design tool has been presented for the triple resonance Tesla transformer of conventional design. The newly derived frequency equation properly accounts for the self-induction and self-capacitance of all the inductors in an orthodox manner. The use of a numerical optimizer to satisfy the constrained frequency equation shows that circuits with arbitrary and precise modal frequency ratios, with circuit parameters fixed or floating, can be easily extracted. The reason that noninteger frequency ratios are of importance is that complete generality of the mathematical tools may be necessary to obtain a procedure for obtaining global maxima in voltage gain of the transformer. The present analysis gives the basic mathematical description of the triple resonance device, in regard to both the frequency and output amplitude equation for the same.

It was decided to examine the amplitude of each voltage wave that aligned in the sample triple resonance transformer. A condensed form of the governing equation for output voltage is written as a voltage gain, and appears in Ref. 12 as

$$
\begin{aligned}
V(t) / V_{0}= & V_{P}\left[C_{\mathrm{I}} \cos \left(2 \pi f_{1} t\right)+C_{\mathrm{II}} \cos \left(2 \pi f_{2} t\right)\right. \\
& \left.+C_{\mathrm{III}} \cos \left(2 \pi f_{3} t\right)\right] .
\end{aligned}
$$

The individual amplitude of each of the three timedependent voltage waves appears as a cosine term within the brackets. Each cosine term has a constant coefficient and 
they are labeled with subscripted roman numerals I, II, and III. Upon inspection of each cosine term and its coefficient an interesting piece of data is revealed. The cosine terms are all very close to the value of \pm 1 , which is expected. There are small differences from \pm 1 due to the finite $1 / 10 \mu$ s time step in the program. But there is a difference between the value of $C_{\mathrm{III}}$ and the values of $C_{\mathrm{I}}$ and $C_{\mathrm{II}}$ (respectively, -0.855 and +1.1). $C_{\mathrm{III}}$ is -0.24 , putting it on the order of $25 \%$ of the other two coefficients (the signs play such that the amplitudes are positive). The high frequency energy is finding it difficult to pass through the device. As a point of interest, if the causes of this design weakness were corrected, and $C_{\mathrm{III}}$ just increased to a value of -1.0 , the voltage gain would increase from $\sim 50$ to $\sim 66$ (if $V_{P}$, the voltage parameter, by some means, remained equal to 22 ).

Since the cubic equation can be manipulated and its shape is controllable; it is probable that new optimal transformers may be discovered. Electrical science has only a few selected pulse generators that can be constructed to operate in the multimegavolt, multimegawatt regime. The transformer is one of these rare devices, and its development may lead to new and unexpected results. As an example, it is not well known, but the transformer generates a nonoscillatory monotonic surge for coupling coefficients in the neighborhood of $0.8 .{ }^{15}$ Which is further invitation to fully characterize the device in both operation and utility.

\section{ACKNOWLEDGMENT}

Gratitude is extended for the knowledge and work of Terry Fritz of Fort Collins, Colorado in regard to the ORCAD PSPICE analysis.

${ }^{1}$ E. A. Abramyam, IEEE Trans. Nucl. Sci. 18, 447 (1970).

${ }^{2}$ G. J. Rohwein, Sandia Laboratories Report No. SAND79-0813, 1979 (unpublished).

${ }^{3}$ N. Tesla, U.S. Patent No. 1,119,732 (1 December 1914).

${ }^{4}$ F. M. Bieniosek, Proceedings of the sixth IEEE Pulsed Power Conference, 1987 (unpublished), p. 700.

${ }^{5}$ F. M. Bieniosek, Rev. Sci. Instrum. 61, 1717 (1990).

${ }^{6}$ William Wysock, Web page at wysock@ttr.com

${ }^{7}$ F. M. Bieniosek, U.S. Patent No. 4,833,421 (23 May 1989).

${ }^{8}$ A. C. M. de Queiroz, 2000 IEEE ISCAS, Geneva, Switzerland, May 2000 (unpublished), Vol. V, pp. 413-416.

${ }^{9}$ Computer code ORCAD PSPICE, ORCAD, 9300 SW Nimbus Ave., Beverton, OR 97008.

${ }^{10}$ See EPAPS Document No. E-RSINAK-77-209603 for the derivation of the frequency equation of the triple resonance Tesla transformer and the computer program for the design of transformers with arbitrary frequency ratios (to include noninteger). This document can be reached via a direct link in the online article's HTML reference section or via the EPAPS homepage (http://www.aip.org/pubservs/epaps.html).

${ }^{11}$ H. G. Jacob, NASA Technical Note D-6978, 1972 (unpublished).

${ }^{12}$ J. R. Reed, Rev. Sci. Instrum. 76, 1 (2005).

${ }^{13}$ L. S. Jacobsen and R. S. Ayre, Engineering Vibrations (McGraw-Hill, New York, 1958), Chaps. 7 and 8.

${ }^{14}$ T. von Karman and M. A. Biot, Mathematical Methods in Engineering (McGraw-Hill, New York, 1940), Chap. 6, Sec. VI.

${ }^{15}$ G. J. Rohwein and M. T. Buttram, Sandia Laboratories Report No. SAND77-0174 (1977); see Chap. III concerning couplings near 1.0. 